Int. J. Dev. Biol. 64: 289-297 (2020)

https://doi.org/10.1387/ijdb.190153ar

\title{
Drosophila Mon1 and Rab7 interact to regulate glutamate receptor levels at the neuromuscular junction
}

\author{
ANAGHA BASARGEKAR ${ }^{1}$, SHWETA YOGI ${ }^{1}$, ZEESHAN MUSHTAQ ${ }^{2}$, SENTHILKUMAR DEIVASIGAMANI ${ }^{3}$, \\ VIMLESH KUMAR ${ }^{2}$, GIRISH S. RATNAPARKHI ${ }^{3}$ and ANURADHA RATNAPARKHI ${ }^{*}, 1$ \\ ${ }^{1}$ Agharkar Research Institute, Pune, ${ }^{2}$ Indian Institute of Science Education and Research, Bhopal and \\ ${ }^{3}$ Indian Institute of Science Education and Research, Pune, India
}

\begin{abstract}
Regulation of post-synaptic receptors plays an important role in determining synaptic strength and plasticity. The Drosophila larval neuromuscular junction $(\mathrm{nmj})$ has been used extensively as a model to understand some of these processes. In this context, we are interested in the role of Drosophila Monensin sensitivity protein 1 (DMon1) in regulating glutamate receptor (GluRIIA) levels at the $\mathrm{nmj}$. DMon 1 is an evolutionarily conserved protein which, in complex with calcium caffeine zinc sensitivity1 (CCZ1), regulates the conversion of early endosomes to late endosomes through recruitment of Rab7. C-terminal deletion mutants of Dmon 1 (Dmon 1481) exhibit lethality. The escapers have a short life span and exhibit severe motor defects. At the $\mathrm{nmj}$, these mutants show defects in synaptic morphology and a strong increase in GluRIIA levels. The mechanism by which Dmon 1 regulates GluRIIA is unclear. In this study, we have characterized an EMS mutant referred to as $\operatorname{pog}^{1}$ and demonstrate it to be an allele of Dmon1. Further, we have examined the role of rab7 in regulating GluRIIA. We show that similar to Dmon1, knock-down of rab7 using RNAi in neurons, but not muscles, leads to an increase in GluRIIA. Loss of one copy each of Dmon1 and rab7 leads to a synergistic increase in receptor expression. Further, overexpression of an activated Rab7 can rescue the GluRIIA phenotype observed in Dmon ${ }^{\Delta 181}$ mutants. Together, these results highlight a neuronal role for Rab7 in GluRIIA regulation and underscore the importance of the endo-lysosomal pathway in this process.
\end{abstract}

KEY WORDS: Dmon1, Rab7, GluRIIA, nmj, Drosophila

\section{Introduction}

Post-synaptic neurotransmitter receptors play an important role in determining synaptic strength and plasticity. Receptor levels are altered in response to changes in neurotransmitter release to maintain homeostasis. The Drosophila larval neuromuscular junction (nmj) has been used for a long time as a model to study synaptic development and homeostasis. These synapses are glutamatergic akin to the central synapses found in the vertebrate CNS. The glutamate receptors at the $\mathrm{nmj}$ are tetramers made up of four subunits: GluRIIA or GluRIIB, GluRIIC, GluRIID and GluRIIE. Receptors carrying the A or the B type subunit differ in their conductance properties and are shown to be reciprocally regulated such that an increase in the levels of GluRIIA leads to corresponding decrease in GluRIIB (Petersen et al., 1997; DiAntonio et al., 1999; Marrus et al., 2004). The mechanisms that regulate glutamate receptor expression, clustering and turn-over are still poorly understood. A classic study by Broadie and Bate more than two decades ago showed that expression of GluRIIA during embryogenesis takes place in two waves. The first wave is autonomous to the muscle and independent of innervation, while the second wave is dependent of innervation. Clustering of the receptors is also dependent on contact between the nerves and muscle (Brodie and Bate, 1993). In the absence of innervation or electrical activity, the muscles fail to upregulate receptor expression in the second wave and clustering fails (Brodie and Bate., 1993). A more recent analysis of the kinetics of GluRIIA transcription suggests that most of the mRNA required for protein synthesis during the

Abbreviations used in this paper: DMon 1, Drosophila monensin sensitivity protein 1; GluRIIA, glutamate receptor subunit; nmj, neuromuscular junction; Rab, Rasrelated in brain.

*Address correspondence to: Anuradha Ratnaparkhi. Agharkar Research Institute, Pune, 411004, India. E-mail: anu.aripune@gmail.com (D) https://orcid.org/0000-0001-6548-6185

Supplementary Material (one figure) for this paper is available at: https://doi.org/10.1387/ijdb.190153ar

Submitted: 30 June, 2019; Accepted: 19 August, 2019.

ISSN: Online 1696-3547, Print 0214-6282

(c) 2020 UPV/EHU Press

Printed in Spain 
embryonic and larval stages is transcribed during embryogenesis with a sharp increase in transcript levels occurring around the time of nerve-muscle contact. An equally sharp decrease in mRNAlevels is seen soon after, with no further increase in mRNA levels. The change in GluRIIA protein levels appears to be more gradual and steadily increases through embryonic and larval stages (Ganesan et al., 2009) suggesting that most of the mRNA synthesized during embryogenesis for use during larval stages is sequestered as ribonucleoparticles for subsequent use.

The molecular nature of the pre-synaptic inputs that regulate post-synaptic receptor expression, clustering and maintenance of these GluRIIA clusters has remained elusive. Recently, Lola was identified as a transcription factor regulating the expression of many post-synaptic components including GluRIIA. Interestingly, Lola was found to be and sensitive to neuronal activity, with increase in stimulation leading to downregulation of Lola activity (Fukui et al., 2012). The molecular identity of other signals, if any, that might function as part of this pre-synaptic regulatory network are not known.

DrosophilaMon1(DMon1) is a conserved endocytic factor which, in complex with CCZ1, helps recruit Rab7 onto endosomes, facilitating the conversion of an early endosome to a late endosome. The protein was first identified in yeast as a factor required for all fusion events to the lysosome (Wang et al., 2002; Wang et al., 2003). This function of recruiting Rab7 appears to be conserved across species from yeast to mammals including plants (Poteryaev et al., 2010; Nordmann et al., 2010; Kinchen and Ravichandran., 2010; Yousefian et al., 2013; Cui et al., 2014). While the cellular function of Mon1 is now well established, its physiological role is only beginning to be addressed. For example, in Arabidopsis, mon 1 mutants show poor male fertility due to delayed tapetal degeneration and programmed cell death (Cui et al., 2017). In Cryptococcus neoformans Mon1 is essential for virulence (Son et al., 2018).

We had previously generated, through P-element excision, a mutation in Dmon1 (Dmon $1^{\Delta 181}$ ), that deletes the C-terminal region of the protein. Dmon $1^{\Delta 181}$ mutants show poor viability and motor abilities. The synaptic morphology in these animals is altered. However, a striking phenotype observed in these animals was the elevated levels of GluRIIA in synaptic, and often, the extrasynaptic regions. The requirement of Dmon1 appears to be primarily neuronal, with neuronal knock-down phenocopying the GluRIIA phenotype and expression of Dmon1 in neurons but not muscle, being able to rescue the mutant lethality (Deivasigamani et al., 2015). The mechanism by which DMon1 regulates GluRIIA levels is not clear. Given the relationship between DMon1 and Rab7, we wondered whether Rab7 might play a role in this process. In this study, we have focused our attention to determining whether DMon1 and Rab7 interact to regulate GluRIIA levels at the larval neuromuscular junction.

Here, we describe a new allele of Dmon1 previously identified and referred to as $\operatorname{pog}^{1}$ (Matthew et al., 2009). Using genetics and sequencing, we demonstrate that $\operatorname{pog}^{1}$ (hereafter referred to as Dmon ${ }^{156}$ ) is an allele of Dmon 1 with a stop codon at residue 157 of the amino acid sequence. Like the C-terminal deletion mutant, Dmon $1^{156 / 4181}$ adults show impaired motor abilities and shortened lifespan. At the synapse these mutants show an increase in GluRIIA levels. To evaluate the role of Rab7 in the regulation of GluRIIA, we have knocked down rab7in neurons using RNAi. Interestingly, while neuronal knock-down leads to an increase in the intensity of
GluRIIA staining, downregulation of $r a b 7$ in the muscle has little effect. Further, there appears to be a dose dependent effect with Rab7 ${ }^{\mathrm{CA}}$ : high levels of expression leads to an increase in GluRIIA.

We show that Dmon1 and rab7 interact to regulate GluRIIA levels. Transheterozygous mutants carrying one copy each of the Dmon $1^{\Delta 181}$ and rab7' mutation show an increase in GluRIIA levels that are comparable to homozygous Dmon $1^{4181}$. Furthermore, expression of Rab7 ${ }^{\mathrm{CA}}$ in a Dmon $1^{\Delta 181}$ mutant background is able to suppress the GluRIIA phenotype suggesting that the two genes are likely to be part of the same regulatory pathway. Our results thus demonstrate a role for a presynaptic DMon1-Rab7 dependent endosomal pathway in regulating post-synaptic receptor levels.

\section{Results}

\section{Identification and characterization of Dmon1 156}

$\operatorname{pog}^{1}$ is an EMS mutant first described as a mutation affecting germband extension during gastrulation in early embryogenesis (Matthew et al., 2009). pog $^{1}$ is homozygous lethal and the mutants fail to survive beyond the second instar larval stage. It shows non-complementation with $D f(2 L) 9062$ indicating that the mutation is likely to be in either Dmon1 or smog- two genes that are uncovered by the deficiency line. We crossed $p o g^{1}$ to Dmon $1^{1181}$ and Dmon $1^{1129}$ mutants: the former carries a deletion restricted to the 3' region of Dmon1, while the latter has a deletion spanning the 3' region of Dmon1 and 5' region of the adjacent smog gene (Deivasigamani et al., 2015). Non-complementation was observed in both cases indicating that the mutation is likely to be in Dmon1. Indeed, sequence analysis of Dmon 1 in pog $^{1}$ showed presence of single base pair change leading to an amber mutation at residue 157 (Fig. 1A), suggesting absence of a full-length protein. We hereafter refer to $\operatorname{pog}^{1}$ as Dmon $1^{156}$.

We have previously shown that homozygous Dmon $1^{\Delta 181}$ escaper adults have a short lifespan and strong motor defects. Consistent with this, both Dmon $1^{156 / \Delta 181}$ and Dmon 156/Df(2L)9062 animals were short-lived and showed poor motor abilities (Fig. $1 \mathrm{~B}, \mathrm{C}$ ). To determine if neuronal expression of UAS-Dmon $1:: H A$ rescues these defects, we expressed the transgene in Dmon1156/A181 mutants and found that both defects could be rescued completely to match wildtype animals (Fig.1 B,C). Interestingly, the lethality in homozygous Dmon $1^{156}$ mutants could not be rescued by expression of UAS-Dmon 1::HA indicating the presence of a second site 'hit' that contributes to lethality in these mutants. However, the rescue of lethality and climbing defect in Dmon $1^{156 / \Delta 181}$ through expression of UAS-Dmon $1:: H A$ supports and validates Dmon $1^{156}$ as an allele of Dmon1.

\section{Characterization of synaptic phenotypes in Dmon1 ${ }^{156}$}

Homozygous Dmon $1^{\Delta 181}$ mutant larvae show defects in synaptic morphology: the boutons tend to be larger and odd shaped with many supernumerary or satellite boutons (Fig 2B,C; Deivasigamani et al., 2015). A similar phenotype was observed in Dmon1 ${ }^{156 / 4181}$ larvae: the boutons were often bigger and, like the other allele, showed many more supernumerary boutons (Fig. 2 D,E) with no change in bouton number.

Homozygous Dmon $1^{\Delta 181}$ mutants show elevated levels of GluRIIA at post-synaptic densities. On an average, the observed increase is nearly two-fold (Deivasigamani et al., 2015). An increase in GluRIIA levels was also observed in Dmon1 ${ }^{156 / \Delta 181}$ animals (Fig. $2 \mathrm{H}-\mathrm{H}^{\prime \prime}$ ). 
However, unlike Dmon $1^{\Delta 181}$ the increase was approximately $30 \%$ (Fig. 2l). The phenotypes associated with synaptic morphology and GluRIIA were suppressed upon expression of UAS-Dmon $1:: H A$, confirming that they are caused due to loss of Dmon1 function (Fig. 2 F,J). This provides further validation to Dmon $1{ }^{156}$ being an allele of Dmon1.

Loss of Dmon1 does not alter quantal size or quantal content Overexpression of GluRIIA increases post-synaptic sensitivity leading to increase in quantal size, which is the response to release of a single neurotransmitter vesicle. These animals also show an increase in the evoked response or evoked junction potential (EJP) but no change in quantal content or the total number of vesicles released (Petersen et al., 1997).

Homozygous Dmon $1^{4181}$ mutants show nearly a 2-fold increase in GluRIIA levels. To determine whether the increase in receptor levels has similar physiological effects as GluRIIA overexpression, we carried out intracellular recordings on homozygous Dmon $1^{\Delta 181}$ and Dmon $1^{\Delta 181} / D f(2 L) 9062$ animals. Consistent with the increase in GluRIIA, in both genotypes, a significant increase in EJP was observed (FIG. 3B-C \& $\mathrm{H}$ ) which was rescued by neuronal expression of UAS-Dmon1::HA (Fig. $3 \mathrm{E}, \mathrm{H}$ ). Surprisingly, we failed to observe any change in quantal size, frequency of the mEPSPs (Fig. 3 F,G) and quantal content (Fig. 3I). The lack of an increase in post-synaptic sensitivity suggests that either many of the GluRIIA positive receptors are non-functional or, that there is compensation possibly due to the decrease in vesicle size and/or the decrease in GluRIIB which has been observed in these mutants (Deivasigamani et al., 2015).

\section{Neuronal knock-down of Rab7 increases GluRIIA}

The above results show that loss of Dmon1 leading to an increase in GluRIIA levels alters neurotransmission. However, the mechanism by which DMon1 regulates receptor levels is not clear. Given that the conserved function of DMon1 is to recruit Rab7, we sought to determine whether the regulation of GluRIIA is dependent

A Sequence of Dmon $1^{156}$ mutant showing the site of nonsense mutation.
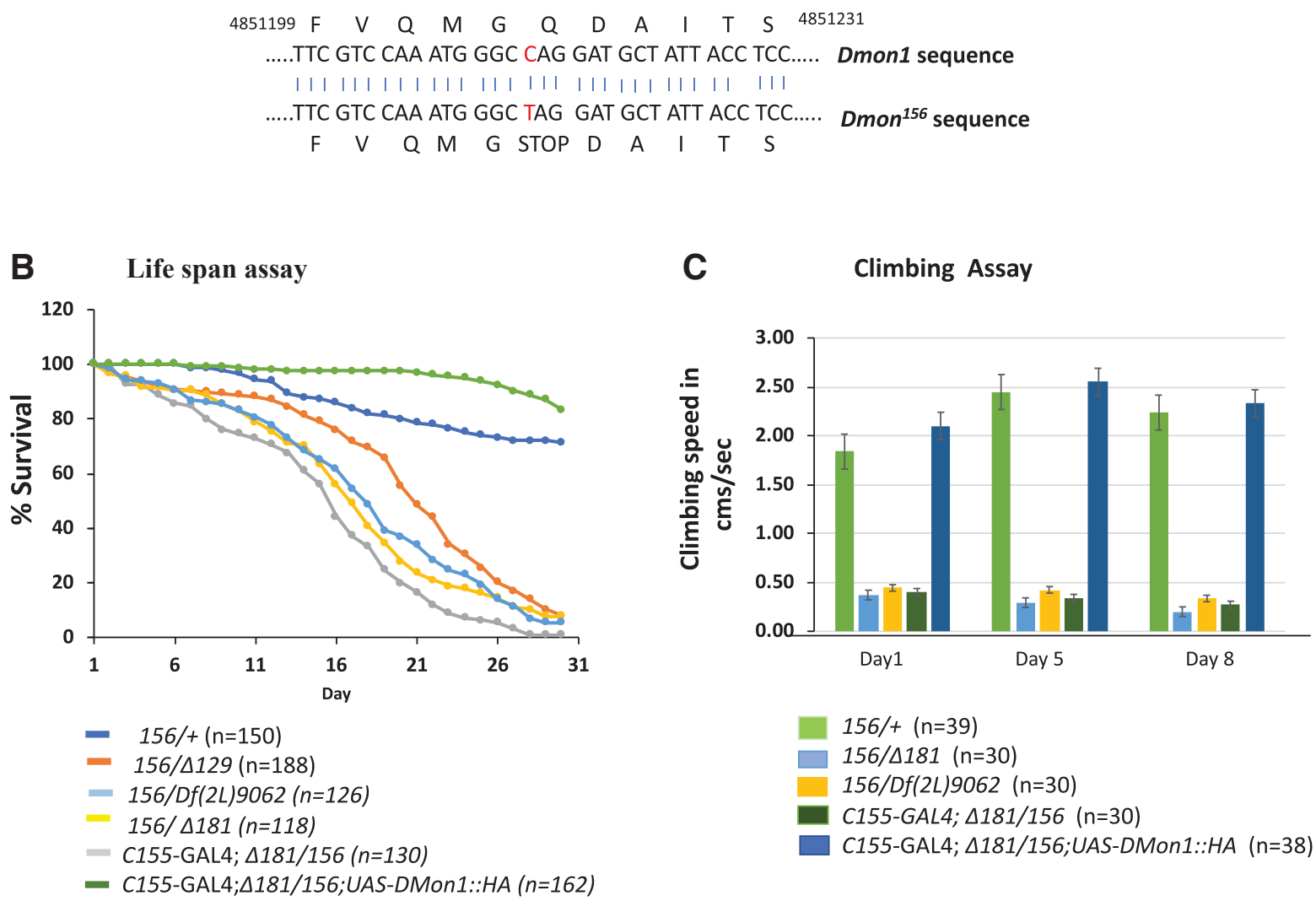

Fig. 1. Identification and characterization of the Dmon 1 156 allele. (A) Shown is the amber mutation in Dmon 1 that generates a stop codon at position 157 of the amino acid sequence. (B) Lifespan of different allelic combinations of Dmon $1^{156}$ mutants. The strongest allelic combination Dmon 1 156/ ${ }^{\Delta 181}$ shows a half-life of approximately 17-18 days. Neuronal expression of UAS-Dmon1::HA rescues life-span to wild-type levels. (C) Shown is a graph of the climbing speed of wildtype and Dmon1 mutants flies at 1, 5 and 8 days post-eclosion. Motor abilities are compromised in Dmon $1^{156 / 4181}$ and Dmon 156/Df(2L)9062 mutants. This is rescued to wild type levels upon neuronal expression of UAS-Dmon1::HA. Climbing speed (in cm/s) on day

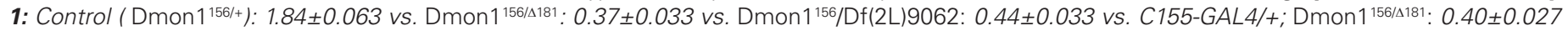

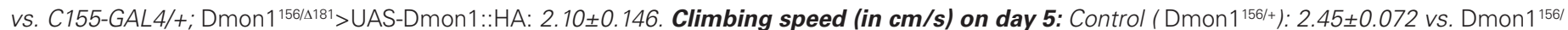

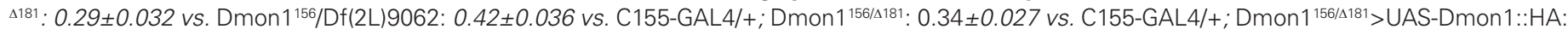

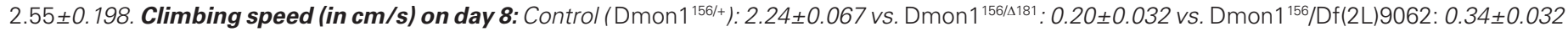

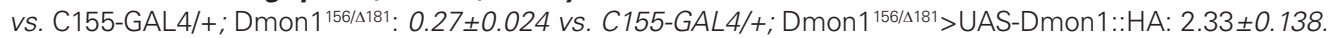


on Rab7. As a first step, we studied the localization of Rab7 at the neuromuscular junction using a genome engineered Rab7 ${ }^{\text {EYFP }}$ line (Dunst et al., 2015) by staining these larvae with anti-HRP and anti-GFP antibodies. Numerous GFP positive puncta were seen distributed all over the muscle and also near boutons in the perisynaptic regions (Fig. 3J). Faint GFP positive puncta were also detected inside boutons suggesting presence of late endosomes in the pre-synaptic compartment (Fig. 3J inset). The puncta were completely absent in Dmon $1^{\Delta 181}$ mutants further reconfirming the role of Dmon1 in recruiting Rab7 onto vesicles (Fig. 3K).

Next, we checked whether knock-down of rab7in neurons alters GluRIIA levels. Expression of UAS-rab7RNAi using D42-GAL4 resulted in a $40 \%$ increase in the intensity of GluRIIA staining (Fig. $3 \mathrm{M}, \mathrm{P})$; An increase of about $30 \%$ was observed with OK6-GAL4 (Fig. 3 O,P). A near two-fold increase in intensity was observed upon expression of the RNAi using a double GAL4 line namely C155-GAL4; elav-GAL4 (Fig. 3P). We examined if knock-down of rab7 in the muscle alters the expression or localization of GluRIIA. Curiously, expression of UAS-rab7RNAiusing C57-GAL4 and mhc-
GAL4 had little effect on GluRIIA levels (Supplementary Figure S1).

Based on the above results, we checked if expression of a constitutively active Rab7 (Rab7 ${ }^{\mathrm{CA}}$ ) leads to a decrease in GluRIIA levels. Interestingly, the effect appeared to be the opposite and dose dependent: while overexpression with D42-GAL4 did not result in any significant change in GluRIIA staining, an increase in receptor levels was observed with C155-GAL4; elav-GAL4 and OK6-GAL4 lines (Fig. 3P)

\section{Mon1 and Rab7 interact to regulate GluRIIA}

The above results indicate that rab7 dependent regulation of GluRIIA is primarily pre-synaptic. To determine whether Dmon1 and rab7 interact to regulate GluRIIA, we examined GluRIIA levels in the trans-heterozygous mutant larvae carrying one copy each of Dmon $1^{1181}$ and rab7'. A significant increase in receptor expression was observed (Fig4. B). Notably, Dmon $1^{4181 /+}$ and rab7 $7^{1 /+}$ larvae did not show any significant change in synaptic GluRIIA levels when compared with wildtype (Fig. 4C). Further, the near two-fold increase in GluRIIA intensity in trans-heterozygous Dmon1 ${ }^{1181 /+}$,

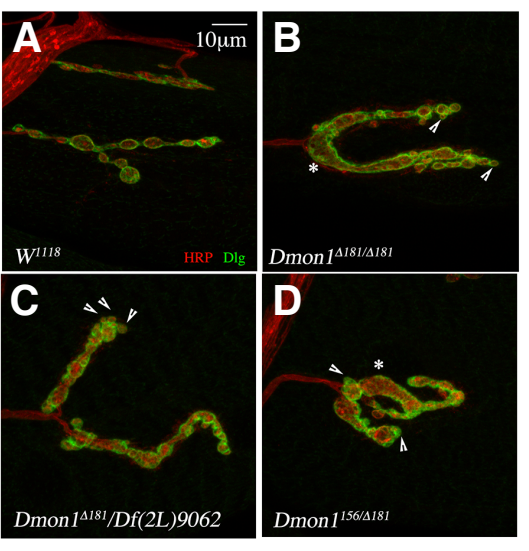

E

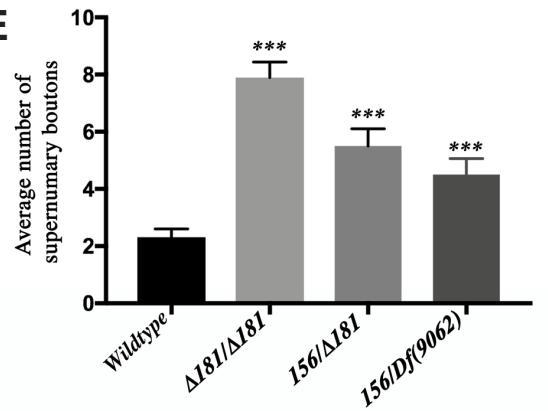

$\mathbf{F}$

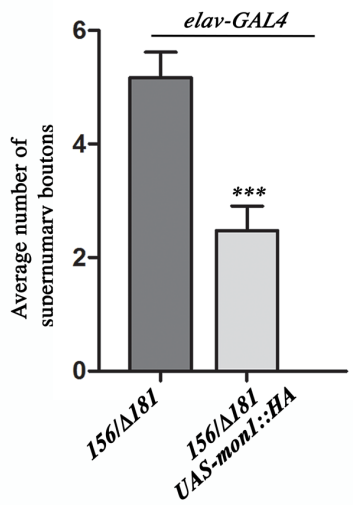

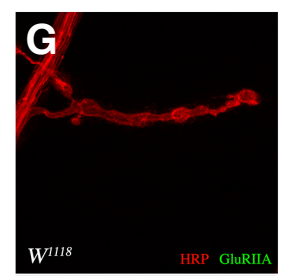
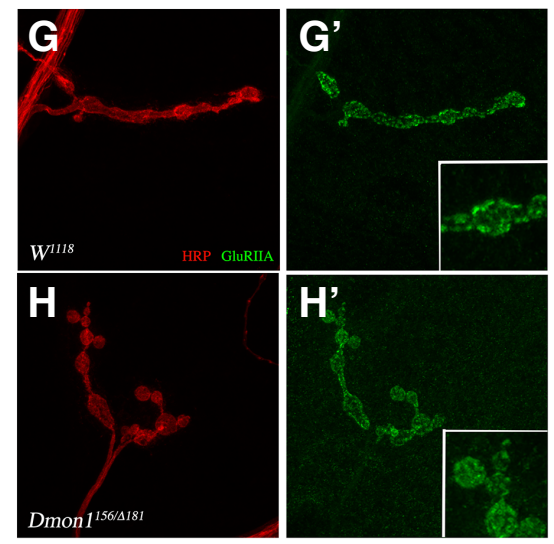

I

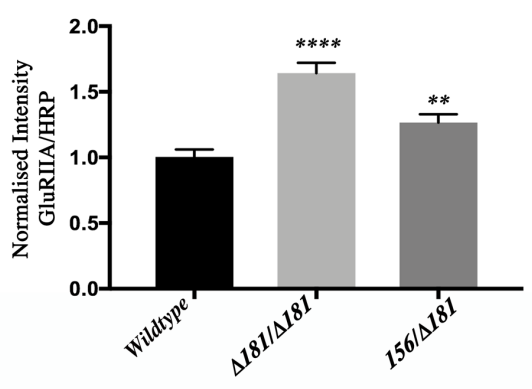

$\mathbf{J}$

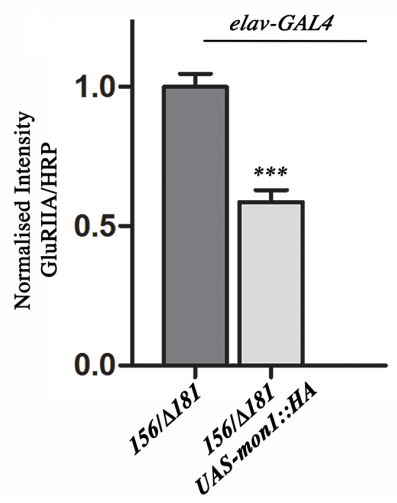

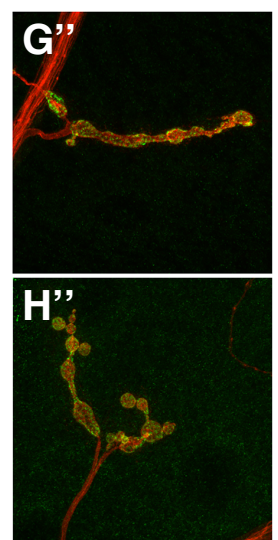

Fig. 2. Characterization of the synaptic phenotype in Dmon $1^{156}$. (A-D) Muscle 4 synapse stained with HRP (red) and Dlg (green). (A) Wildtype $\left(\mathrm{w}^{1118}\right)$ synapse. Note the pearl-on-a-string arrangement of boutons. (B,C) Synapse of a homozygous Dmon $1^{\Delta 181}$ (B) and Dmon1 $^{\Delta 181}$ Df(2L)9062 (C) larva. Note presence of fused and irregularly shaped bouton (asterisk) and satellite boutons (arrowheads). (D) Dmon1 156/4181 larvae exhibit a similar synaptic morphology with irregularly shaped boutons and satellite or supernumerary boutons. (E) Quantification of the average number of satellite boutons per synapse in different alleles of Dmon1. A significant increase in satellite boutons is seen in the mutants $\left(\mathrm{w}^{1118}\right.$ : $2.31 \pm 0.286, n=19$; homozygous Dmon $1^{\Delta 181}$ : $7.89 \pm 0.54, n=19$; Dmon 1 ${ }^{156 / \Delta 181}: 5.5 \pm 0.6, n=18$; Dmon1 156/Df(2L)9062: 4.504 $\pm 0.562, n=16)$. (F) The satellite bouton phenotype in Dmon $1^{156 / \Delta 181}$ is suppressed by expression of UAS-Dmon $1:: H A$

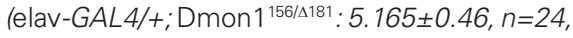
elav-GAL4/+; Dmon1 ${ }^{156 / \Delta 181}>$ UAS-Dmon1::HA $2.472 \pm 0.43, n=23)$. (G,H) A muscle 4 synapse from $\mathrm{W}^{1118}(\mathbf{G})$ and Dmon1 ${ }^{156 / \Delta 181}$ (H) stained with HRP (red) and GluRIIA (green). Note the increase in intensity of GluRIIA. (I) Quantification of the intensity of GluRIIA in wildtype $(1 \pm 0.056, n=16)$, homozygous Dmon $1^{4181}(1.744 \pm 0.085, n=17)$ and Dmon1 ${ }^{156 /}$ $\Delta 181(1.292 \pm 0.065, n=20)$ animals. The latter shows a $30 \%$ increase in GluRIIA intensity compared to a near $70 \%$ increase in homozygous Dmon $1^{\Delta 181}$. (J) Neuronal expression of UAS-Dmon 1::HA rescues the GluRIIA phenotype in Dmon1 ${ }^{156 / \Delta 181}$ mutants: $1 \pm 0.047$ (elav-GAL4/+, Dmon 1156/4181, $n=24$ ) vs. $0.58 \pm 0.042$ (elav-GAL4/+, Dmon $^{156 / \Delta 181}>$ UAS-Dmon $\left.1:: H A, n=22\right) . \quad * * *$ indicates $P<0.0001 ; * *$ indicates $P<0.001$; ${ }^{*}$ indicates $P<0.01 ; n$, number of synapses. 


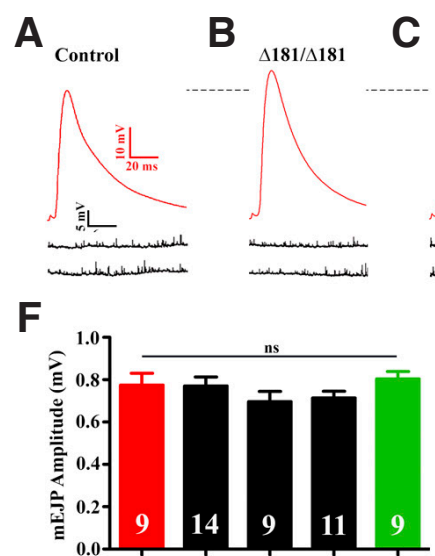

$\mathrm{H}$
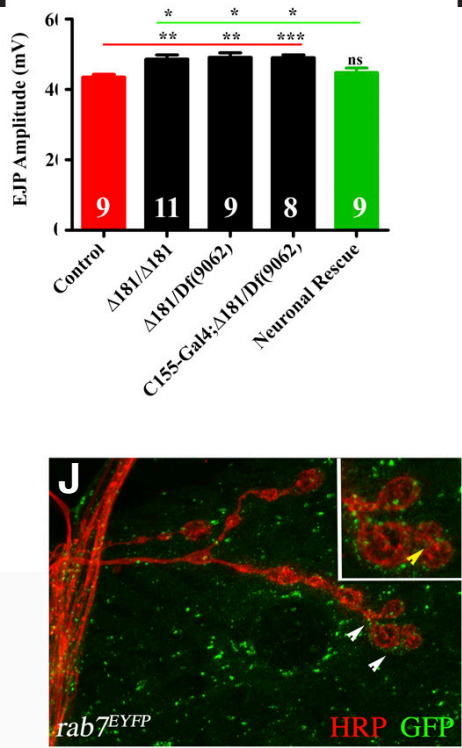

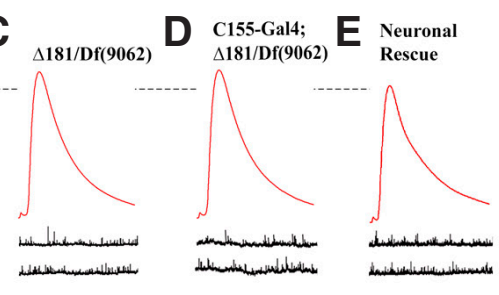

G
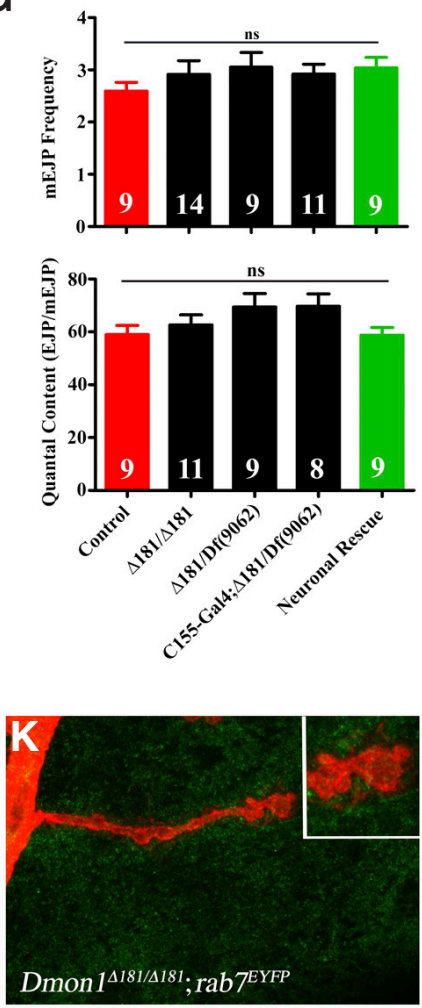
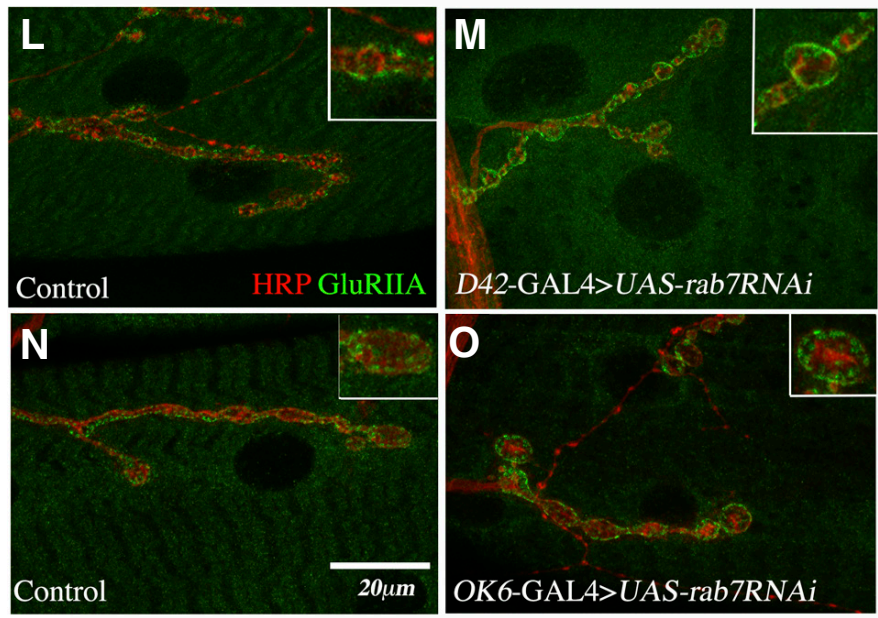

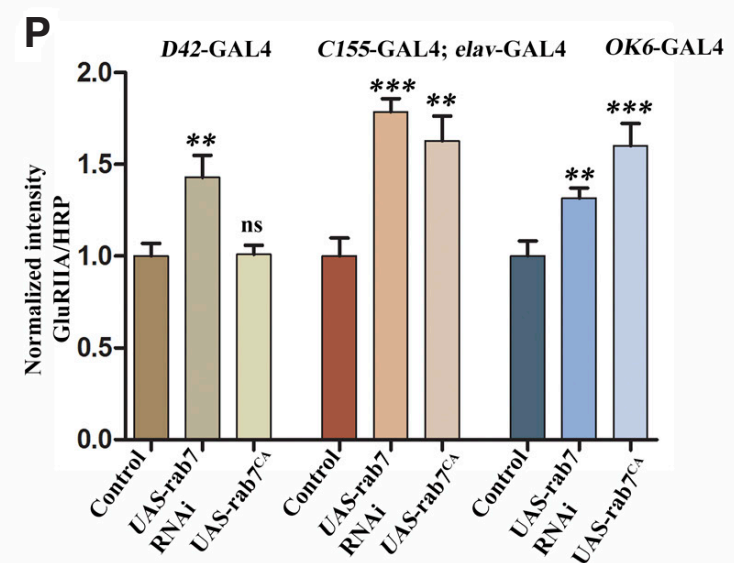

Fig. 3. Neuronal downregulation of rab7 increases GluRIIA levels. (A-E) Representative traces of evoked (Red) and spontaneous (Black) responses of indicated genotypes. Recordings were carried out in $1.5 \mathrm{mM} \mathrm{Ca}{ }^{2+}$ containing $\mathrm{HL} 3$ and nerves innervating muscle 6/7 were stimulated at $1 \mathrm{~Hz}$ to

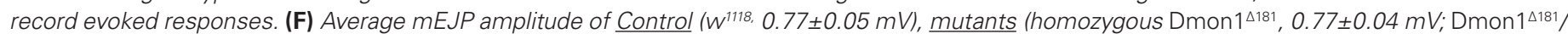
$\mathrm{Df}(2 \mathrm{~L}) 9062,0.70 \pm 0.05 \mathrm{mV}$ ), control for rescue (C155-GAL4/+; Dmon14181/Df(2L)9062, $0.71 \pm 0.03 \mathrm{mV}$ ) and rescued mutants (C155-GAL4/+; Dmon1 ${ }^{181 /}$ Df(2L)9062; UAS-Dmon1::HA, $0.80 \pm 0.03 \mathrm{mV}$ ). (G) Average mEJP frequency of Control (w ${ }^{1118}, 2.59 \pm 0.16 \mathrm{~Hz}^{2}$, mutants (homozygous Dmon1 $18181,2.91 \pm$ $0.25 \mathrm{~Hz}$; Dmon1 181/Df(2L)9062, $3.05 \pm 0.27 \mathrm{~Hz}$ ), control for rescue (C155-GAL4/+; Dmon1 18181/Df(2L)9062, $2.92 \pm 0.18 \mathrm{~Hz})$ and rescued mutants (C155-

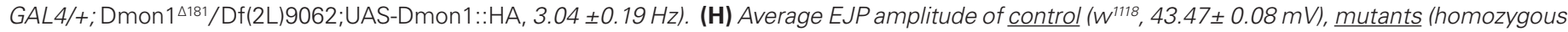
Dmon1 1181, $48.64 \pm 1.17 \mathrm{mV}$; Dmon1 1181 Df(2L) 9062, $49.12 \pm 1.33 \mathrm{mV})$, control for rescue (C155-GAL4/+; Dmon1 $181 / \mathrm{Df}(2 \mathrm{~L}) 9062,49.05 \pm 0.77 \mathrm{mV})$

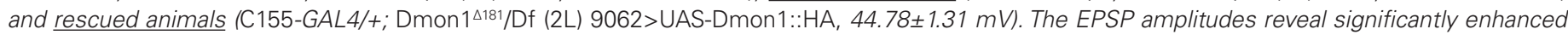
evoked potentials in Dmon1 alleles, which are rescued by expression of UAS-Dmon 1::HA in the mutants. (I) Average quantal content of control (W ${ }^{1118}$, $58.97 \pm 3.45$ ), mutants (homozygous Dmon1 ${ }^{\Delta 181}, 62.67 \pm 3.76$; Dmon1 ${ }^{\Delta 181}$ Df(2L) 9062, $69.46 \pm 5.07$ ), control for rescue (C155-GAL4/+; Dmon $1^{\Delta 181 / D f}$

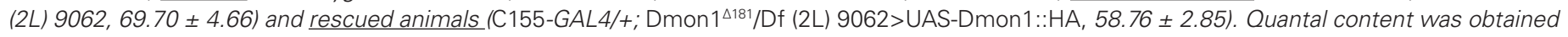
by dividing average EJP amplitude with average mEJP amplitude of individual recordings. (J,K) Rab7EYFP line stained with anti-HRP and anti-GFP (J). Rab7 ${ }^{E Y F P}$ in homozygous Dmon1 ${ }^{\triangle 181}$ mutants (K). Green puncta in (J) shows localization of Rab7:EYFP. Note the distribution of Rab7 positive endosomes in the muscle. Inset shows presence of Rab7 positive puncta in the pre-synaptic compartment and perisynaptic regions. The puncta are completely absent in homozygous Dmon1 ${ }^{\Delta 181}$ mutants. (L-O) Representative images of the effect of downregulation of rab7 on GluRIIA levels using D42-GAL4 $(\mathbf{L}, \mathbf{M})$ and OK6-GAL4 (N,O). Note the increase in receptor levels ( $M$ and $O$, respectively). (P) Change in staining intensity of GluRIIA upon expression of UAS-rab7RNAi and UAS-rab 7 CA with different drivers: D42-GAL4: Control (D42-GAL4/+; $1 \pm 0.056, n=14)$ vs. UAS-rab7RNAi (D42-GAL4> UASrab7RNAi, $1.430 \pm 0.119, n=13)$ vs. UAS-rab7CA (D42-GAL4> UAS-rab7CA, $1.009 \pm 0.049, n=9) ;$ C155-GAL4; elav-GAL4: Control (C155-GAL4/+; elav-GAL4/+, $1 \pm$ $0.099, n=10)$ vs. UAS-rab7RNAi (C155-GAL4; elav-GAL4> UAS-rab7RNAi, $1.785 \pm 0.072, n=20)$ vs. UAS-rab7cA $(1.628 \pm 0.134, n=12)$ and Control (OK6GAL4/+, $1 \pm 0.082, n=18)$ vs. UASrab7RNAi (OK6-GAL4> UAS-rab7RNAi, $1.316 \pm 0.054, n=20)$ vs. UAS-rab7CA (OK6-GAL4> UAS-rab7CA, $1.602 \pm 0.12$, $n=19 .{ }^{* *}$ indicates $P<0.0001 ; *{ }^{*}$ indicates $P<0.001 ;{ }^{*}$ indicates $P<0.01 ; n$, number of synapses. (F,G, $\left.\mathbf{H}, \mathbf{I}\right)$ The numbers in the bars represent number of animals used for recordings and/or quantification. Error bars represent standard error of the mean (SEM). Statistical analysis based on two-tailed Student's t-test. ${ }^{*} P \leq 0.05,{ }^{*} P \leq 0.01$. ${ }^{*} * P \leq 0.001$. 

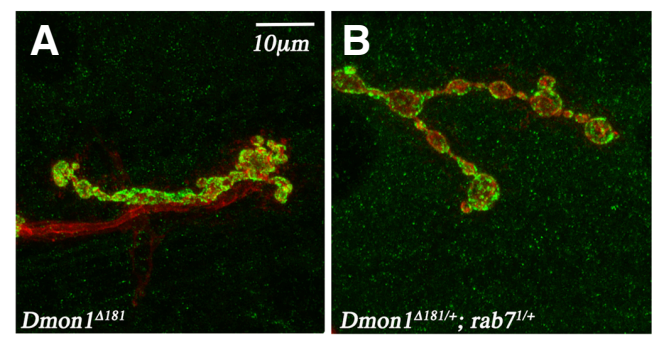

C

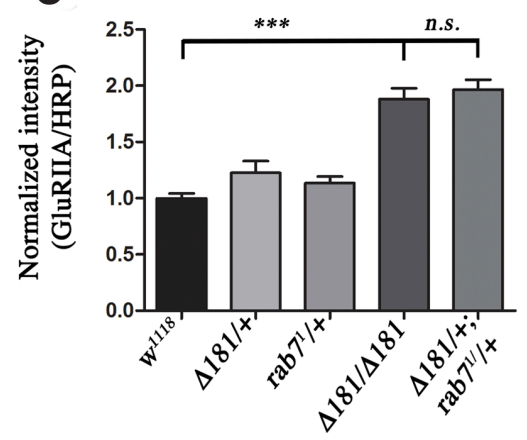

D

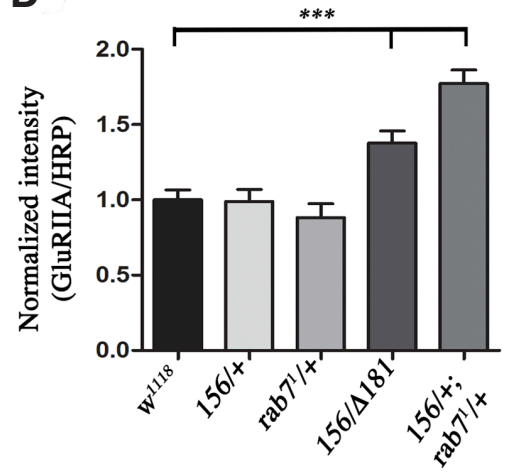

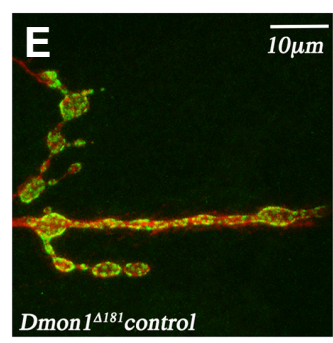

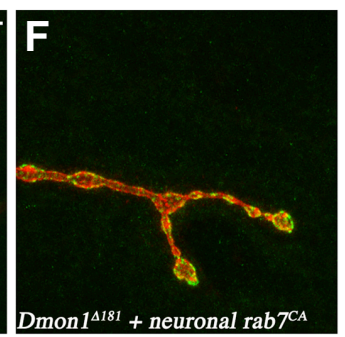

G

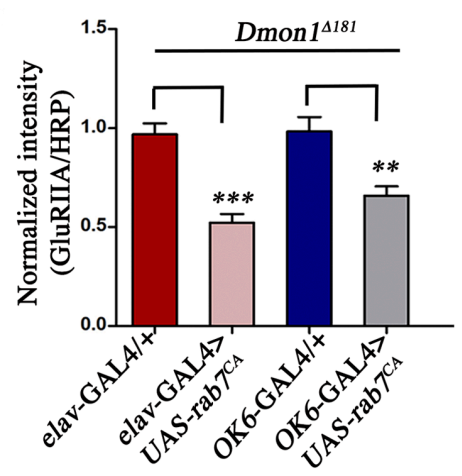

H

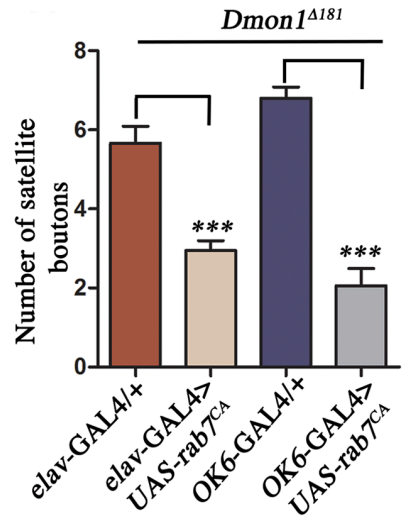

Fig. 4. Dmon 1 and rab71 interact in a synergistic manner to regulate GluRIIA levels. (A,B) Homozygous Dmon $1^{\Delta 181}(A)$ and $D m o n 1^{4181 /+}$; rab7 $7^{1 /+}(B)$ stained with anti-HRP (red) and anti-GluRIIA (green) show comparable levels of GluRIIA. (C) Graph showing normalized values for intensity of GluRIIA:HRP

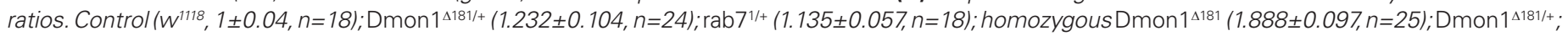
rab7 ${ }^{1 /+}(1.965 \pm 0.088, n=32)$. The increase in GluRIIA in trans-heterozygous mutant animals is comparable to homozygous Dmon $1^{\Delta 181}$. (D) Graph showing the normalized values for intensity of GluRIIA:HRP ratios. Control ( $\left.w^{1118}, 1 \pm 0.06, n=20\right)$; Dmon1 156/+ $(0.99 \pm 0.076, n=20)$; rab71/+ $(0.88 \pm 0.091, n=20)$; Dmon1 ${ }^{156 / \Delta 181}(1.37 \pm 0.08, n=19)$; Dmon1 ${ }^{156 /+} ;$ rab7 $^{1 /+}(1.777 \pm 0.09, n=19)$. (E,F) Expression of UAS-rab7CA in homozygous Dmon $1^{\Delta 181}$ mutants rescues the synaptic and GluRIIA phenotype associated with the mutants. (G) Normalized values for intensity of GluRIIA:HRP ratios. Mutant control (Dmon $1^{\Delta 181 ;}$

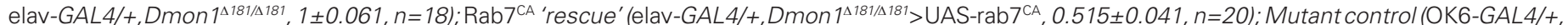
Dmon14181/4181, $1 \pm 0.071, n=20)$; rab7CA 'rescue' (OK6-GAL4/+, Dmon14181/481 >UAS-rab7CA, 0.685 $\pm 0.051, n=20)$. (H) Expression of UAS-rab7CA rescues

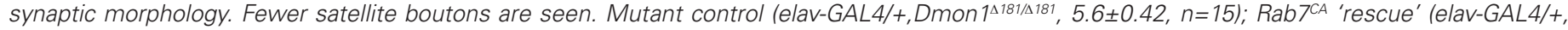

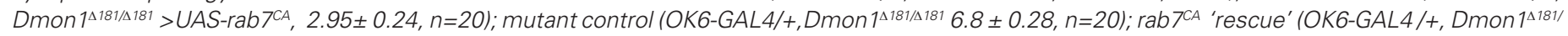
$\triangle 181>$ UAS-rab7CA $, 2.05 \pm 0.43, n=18) .{ }^{* * *}$ indicates $P<0.0001,{ }^{* *}$ indicates $P<0.001$ and ${ }^{*}$ indicates $P<0.01$. $n$, number of synapses.

rab7 $7^{1 /+}$ mutants was comparable to the receptor levels observed in Dmon $1^{1181}$, indicating that Dmon1 and rab7 interact in a dose dependent manner to regulate GluRIIA. The increase in receptor expression was observed not only at the synapse, but in extrasynaptic regions as well which is often seen in Dmon $1^{\Delta 181}$ mutants (Fig. 4B). A similar synergistic increase in receptor expression was also seen in Dmon $1^{156 /+}$; rab $7^{1 /+}$ animals (Fig.4D). It is to be noted that while the steep increase in receptor levels is comparable to Dmon $1^{\Delta 181}$ mutants, the synaptic morphology in these trans-heterozygous animals is very distinct: the boutons though bigger, are well-spaced with fewer satellites (Fig. 4B).

To further confirm the genetic interaction between Dmon1 and rab7 and to test if they function as part of the same pathway we expressed UAS-rab7CA in homozygous Dmon $1^{\Delta 181}$ mutants to determine if it reduced GluRIIA levels. Indeed, a strong decrease in GluRIIA staining was observed in these animals (Fig. 4 F,G) with fewer number of satellite boutons (Fig. $4 \mathrm{H}$ ). Together these results indicate that Dmon 1 and rab7 interact in the pre-synaptic compartment to regulate GluRIIA at the larval neuromuscular junction. 


\section{Discussion}

Rab proteins play a crucial role in regulating intracellular trafficking by directing endosomes to their appropriate destination. Their dysfunction is associated with various neurodegenerative disease and cancers (Stenmark,H., 2009; Wandinger-Ness and Zerial., 2014; Banworth et al., 2018). Rab7 marks late endosomes and regulates the trafficking and fusion of vesicles with the lysosome. The processes regulated by Rab7 are diverse and range from retrograde trafficking, lysosome positioning, regulation of protein kinases through effectors etc many of which have implications in neurodegenerative disorders (Deinhart et al., 2006; Dodson et al., 2012; Stroupe, C., 2018;). Mutations in Rab7 are associated with Charcot-Marie-Tooth disease 2b (CMT2B) - a type of neurodegenerative disorder that causes axonal degeneration (Verhoeven et al., 2003).

The Mon1-CCZ1 complex functions as a GEF for Rab7 and is essential for its recruitment onto endosomes (Nordmann et al.,2010; Poteryaev et al., 2010). Therefore, it is of interest to determine the extent to which the phenotypes associated with loss of Dmon1 are dependent on rab7. This would be helpful in identifying and understanding Rab7 independent functions of DMon1, if any. We have addressed this in the context of the Drosophila neuromuscular junction where Dmon1, through a pre-synaptic mechanism, regulates GluRIIA levels at the synapse.

We find that presynaptic knock-down of rab7 leads to a significant increase in GluRIIA levels which is comparable to that seen upon knock-down of Dmon1. Further, trans-heterozygous mutants Dmon $1^{\Delta 181 /+} ; \mathrm{rab}^{1 /+}$ and Dmon $1^{156 /+} ; \mathrm{rab}^{1 /+}$ mutants show a dramatic increase in GluRIIA indicating that the two genes interact to regulate GluRIIA. Further, expression of a constitutively active Rab7 (Rab7CA) suppresses not only the defects in synaptic morphology but also the increase in GluRIIA in Dmon1 mutants.

Our results indicate that neuronal and not muscle knock-down of rab7, leads to an increase in the intensity of GluRIIA staining. This is in variance with a study by Lee and colleagues (Lee et al., 2013) involving knock-down of tbc1D15-17-the Drosophila ortholog of the mammalian Rab7 GAP, namely tbc1D15. RNAi mediated knock-down of $t b c 1 D 15-17$ in the muscle but not neuron is seen to affect GluRIIA levels. However, it is to be noted that the effect of rab7 RNAi and rab7CA on GluRIIA has not been evaluated in this study. Moreover, little is known about Rab7 regulation in Drosophila and it is possible that the GAP protein regulating Rab7 in the neuron and muscles is different. These questions will need to be addressed in future studies.

The results from overexpression of $r a b 7^{C A}$ are a little puzzling (Fig. 3). It is not clear why expression with C155-GAL4; elav-GAL4 leads to an increase in GluRIIA staining. In a recent study JiminezOrgaz and colleagues show that TBC1D5 - a GAP for Rab7, in complex with the retromer, controls the localization and cycling of Rab7 such that in the absence of the GAP, the GTP locked form of Rab7 accumulates on the lysosome resulting in defects associated with the non-lysosomal functions of Rab7 (Jimenez-Orgaz et al., 2017). Expression of a constitutively active form of Rab7 showed a similar effect due to accumulation and poor turn-over from the lysosomal membrane due to loss of nucleotide cycling. Given the importance of nucleotide cycling in Rab function, it is possible that expression of Rab7 ${ }^{\mathrm{CA}}$ beyond a threshold results in sequestration of the activated form on membranes resulting in a rab7 loss-of-function like phenotype. This will need to be tested.

The mutation in Dmon $1^{156}$ is in the longin domain. The increase in intensity of GluRIIA in Dmon $1^{156 / \Delta 181}$ is seen to approximately $30 \%$ compared to the nearly two-fold increase seen in Dmon $1^{1181}$ mutants. We do not completely understand the reason for this variation amongst the alleles. It would be interesting to determine functional contribution of the different domains to regulation of GluRIIA levels.

At this point, the precise manner in which DMon1-Rab7 axis regulates GluRIIA is not clear. An interesting point to be noted in this context is that DMon1 is released from the pre-synaptic compartment (Deivasigamani et al., 2015). While we cannot completely rule out the possibility of DMon1 and Rab7 having independent effects on GluRIIA, the evidence based on the genetic interaction between the two, and localization of DMon1 suggests that the DMon1-Rab7 complex probably regulates a secretory process that influences GluRIIA levels. At the nmj, signaling molecules such as wingless and synaptotagmin-4 are known to be released via exosomes (Korkut et al., 2009; Korkut et al., 2013) which arise from multivesicular bodies or late endosomes. It would be interesting to test if the regulation of GluRIIA via the DMon1-Rab7 axis involves such a mechanism.

It is intriguing that post-synaptic knock-down of rab7 does not affect GluRIIA levels in any significant manner. This is somewhat unexpected since in mammalian synapses, endo-lysosomal pathways are known to control the trafficking and turn-over of AMPA receptors (Fernandez-Monreal et al., 2012; Hausser and Schlett., 2017). While the mechanisms regulating GluRIIA turnover at the Drosophila larval $\mathrm{nmj}$ are still poorly characterized, the absence of an effect with UAS-rab7RNAi would imply existence of independent pathways. The other possibility could be that knock-down of Rab7 in the muscle is not sufficient to elicit a phenotype. These possibilities will need to be explored in greater detail. In summary, our findings here highlight a novel role for the neuronal endo-lysosomal pathway in regulating post-synaptic GluRIIA levels, the details of which will need to be elucidated in future studies.

\section{Materials and Methods}

\section{Flystocks}

All stocks were maintained on regular cornmeal agar medium. pog (Kind gift from S. Kerridge); Dmon $1^{1181}$ (Deivasigamani et al., 2015); The following stocks were obtained from the Bloomington Stock Centre: UASRab7RNAi (\#27051); UAS-Rab7Q67L::YFP (\#9779); D42-GAL4 (Rab7 EYFP (\#62545); D42-GAL4 (\#8816); OK6-GAL4 (\#64199). Rab71 (Kind gift from the Juhász lab; Hegedus et al., 2016.)

\section{Molecular mapping and behavioral characterization of Dmon1 ${ }^{156}$}

$D m o n 1^{156}$, previously referred to as pog ${ }^{1}$ (Matthew et al., 2009), does not complement Dmon $1^{\Delta 181}$ indicating that the mutation is likely in Dmon1. To identify the EMS mutation, we PCR amplified and sequenced the entire Dmon 1 gene from $D m o n 1^{156}$. A single base pair change ( $\mathrm{C}$ to $\mathrm{T}$ ) at position 4851219 of the flybase sequence was results in an amber mutation (CAG to TAG) at position 157 of the amino acid sequence of DMon1. The lifespan and motor assays to characterize Dmon $1{ }^{156}$ phenotype was carried out as described in Deivasigamani et al., 2015.

\section{Immunohistochemistry and imaging}

Larval fillets were fixed with Bouins for 15 minutes at room temperature $\left(23-24^{\circ} \mathrm{C}\right)$ and stained using standard protocols (Patel et al., 1994). 
2\% BSA was used for blocking. Anti-HRP (Sigma, 1:1000); anti-GluRIIA (concentrate; DSHB, lowa state, 1:200); anti-GFP (Thermo Fisher, 1:1000). Imaging was done using a Leica SP8 confocal system. All images were captured with a $63 \mathrm{X}, 1.4$ N.A. objective. Image analysis was carried out using ImageJ (NIH) or FIJI software. For measurement of GluRIIA intensity, all genotypes of a set were stained simultaneously. Each time, a cocktail of the primary and secondary antibodies was made and distributed equally to all the genotypes. Imaging was carried out using identical conditions of gain and laser power. Intensity measurements for GluRIIA were carried out as described in Menon et al., 2004 and Deivasigamani et al., 2015.

\section{Electrophysiology}

Intracellular electrophysiology recordings were performed as described previously (Chodhury et al., 2016). Briefly third instar wandering larvae were dissected in modified $\mathrm{HL} 3$ saline containing $70 \mathrm{mM} \mathrm{NaCl}, 5 \mathrm{mM} \mathrm{KCl}$, $20 \mathrm{mM} \mathrm{MgCl} 2,10 \mathrm{mM} \mathrm{NaHCO}, 115 \mathrm{mM}$ sucrose, $5 \mathrm{mM}$ trehalose, $5 \mathrm{mM}$ 4-(2-hydroxyethyl)-1-piperazineethanesulfonic acid (HEPES), and $1 \mathrm{mM}$ EGTA at pH 7.2. For recordings the EGTA was replaced with $1.5 \mathrm{mM} \mathrm{CaCl} 2$ in modified HL3 saline (Verstreken et al., 2002). mEPSPs were recorded for $60 \mathrm{~S}$ in absence of any stimulation. For evoked responses (EPSPs), motor axons were stimulated at $1 \mathrm{~Hz}$ and responses were recorded for 1 minute. All the recordings were made using sharp glass microelectrodes with 15-25 $\mathrm{M} \Omega$ resistance from muscle 6 of $\mathrm{A} 2$ hemi-segment. Data was analysed using an offline software minianalysis (Synaptosoft)

Statistical Analysis. Analysis was done using GraphPad Prism software. Two-tailed student's t-test and ANOVA was used for analysis. Graph in Fig. 4D represents pooled data from 3 independent experiments. Each set contained all genotypes, stained simultaneously and imaged using identical conditions. All values represented in the figures are mean \pm s.e.m.

\section{Acknowledgements}

We thank the Bloomington Drosophila Stock Centre (Indiana), and the Developmental Studies Hybridoma Bank (lowa) for flystocks and antibodies respectively. This work was supported by funds from DBT (BT/PR23318/ $B R B / 10 / 1597 / 2017)$ to $A R$ and GR; GR acknowledges intramural support from IISER-Pune; AB was supported by a CSIR-Senior Research Fellowship.

\section{References}

BANWORTH MJ, LI G (2018). Consequences of Rab GTPase dysfunction in genetic or acquired human diseases. Small GTPases 9: 158-181.

BROADIE K, BATE M (1993). Innervation directs receptor synthesis and localization in Drosophila embryo synaptogenesis. Nature 28;361: 350-353.

CHOUDHURYSD, MUSHTAQZ, REDDY-ALLAS, BALAKRISHNANSS, THAKURRS,

KRISHNAN KS, RAGHU P, RAMASWAMI M, KUMAR V (2016). б2- Adaptin acilitates basal synaptic transmission and is required for regenerating endo-exo cycling pool under highfrequency nerve stimulation in Drosophila. Genetics 203: 369-385.

CUI Y, ZHAO Q, GAO C, DING Y, ZENG Y, UEDA T, NAKANO A, JIANG L (2014). Activation of the Rab7 GTPase by the MON1-CCZ1 Complex Is Essential for PVCto-Vacuole Trafficking and Plant Growth in Arabidopsis. Plant Cell. 26: 2080-2097.

CUI Y, ZHAO Q, XIE HT, WONG WS, WANG X, GAO C, DING Y, TAN Y, UEDA T, ZHANG Y, JIANG L (2017). Monensin sensitivity1 (mon1)/calcium caffeine zinc sensitivity1 (ccz1)-Mediated Rab7 Activation Regulates Tapetal Programmed Cell Death and Pollen Development. Plant Physiol 173: 206-218.

DEINHARDT K, SALINAS S, VERASTEGUI C, WATSON R, WORTHD, HANRAHAN S, BUCCI C, SCHIAVO G (2006). Rab5 and Rab7 control endocytic sorting along the axonal retrograde transport pathway. Neuron.52: 293-305.

DEIVASIGAMANI S, BASARGEKARA, SHWETAK, SONAVANE P, RATNAPARKHI GS, RATNAPARKHI A (2015). A Presynaptic Regulatory System Acts Transsynaptically via Mon1 to Regulate Glutamate Receptor Levels in Drosophila. Genetics 201: 651-664.

DIANTONIO, A., S. A. PETERSEN, M. HECKMANN AND C. S. GOODMAN (1999) Glutamate receptor expression regulates quantal size and quantal content at the Drosophila neuromuscular junction. J Neurosci 19: 3023-3032.

DODSON MW, ZHANG T, JIANG C, CHEN S, GUO M (2012). Roles of the Dro- sophila LRRK2 homolog in Rab7-dependent lysosomal positioning. Hum Mol Genet 21: 1350-1363.

FERNANDEZ-MONREAL, M., T. C. BROWN, M. ROYO AND J. A. ESTEBAN (2012). The balance between receptor recycling and trafficking toward lysosomes determines synaptic strength during long-term depression. J Neurosci32: 13200-13205.

FUKUI A1, INAKI M, TONOE G, HAMATANI H, HOMMA M, MORIMOTO T, ABURATANI H, NOSE A (2012). Lola regulates glutamate receptor expression at the Drosophila neuromuscular junction. Biol Open 1: 362-375.

GANESAN, S., J. E. KARRAND D. E. FEATHERSTONE (2011). Drosophilaglutamate receptor mRNA expression and mRNP particles. RNA Biol 8: 771-781.

HAUSSER A, SCHLETT K (2017). Coordination of AMPA receptor trafficking by Rab GTPases. Small GTPases 19: 1-14.

HEGEDŰS K, TAKÁTS S, BODA A, JIPA A, NAGY P, VARGA K, KOVÁCS AL, JUHÁSZ G (2016). The Ccz1-Mon1-Rab7 module and Rab5 control distinct steps of autophagy. Mol Biol Cell. 27: 3132-3142.

JIMENEZ-ORGAZA, KVAINICKAS A, NÄGELE H, DENNER J, EIMER S, DENGJEL J, STEINBERG $F$ (2017). Control of RAB7 activity and localization through the retromer-TBC1D5 complex enables RAB7-dependent mitophagy. EMBO J 37 : 235-254.

KINCHEN, J. M., AND K. S. RAVICHANDRAN (2010). Identification of two evolutionarily conserved genes regulating processing of engulfed apoptotic cells. Nature 464: 778-782.

KORKUTC, ATAMANB, RAMACHANDRAN P, ASHLEY J, BARRIAR, GHERBESIN, BUDNIK V (2009). Trans-synaptic transmission of vesicular Wnt signals through Evi/Wntless. Cell 139: 393-404.

KORKUT C, LI Y, KOLES K, BREWER C, ASHLEY J, YOSHIHARA M, BUDNIK V (2013). Regulation of postsynaptic retrograde signaling by presynaptic exosome release. Neuron 77: 1039-1046.

LEE MJ, JANG S, NAHM M, YOON JH, LEE S (2013). Tbc1d15-17 regulates synaptic development at the Drosophila neuromuscular junction. Mol Cells. 36: 163-168.

MARRUS, S. B., S. L. PORTMAN, M. J. ALLEN, K. G. MOFFAT AND A. DIANTONIO (2004). Differential localization of glutamate receptor subunits at the Drosophila neuromuscular junction. J Neurosci 24: 1406-1415

MATHEW SJ, KERRIDGE S, LEPTIN M (2009). A small genomic region containing several loci required for gastrulation in Drosophila. PLoS One 4: e7437.

MENON KP, SANYAL S, HABARA Y, SANCHEZ R, WHARTON RP, RAMASWAMI M, ZINN K (2004). The translational repressor Pumilio regulates presynaptic morphology and controls postsynaptic accumulation of translation factor elF-4E. Neuron 44: 663-676.

NORDMANN, M., M. CABRERA, A. PERZ, C. BROCKER, C. OSTROWICZ ENGELBRECHT-VANDRÉ S, UNGERMANN C (2010). The Mon1-Ccz1 complex is the GEF of the late endosomal Rab7 homolog Ypt7. Curr Biol 20: 1654-1659.

PATEL NH (1994). Imaging neuronal subsets and other cell types in whole mount Drosophila embryos and larvae using antibody probes. In Methods in Cell Biology (Ed. Lawrence S.B. Goldstein and Eric. A. Fyrberg), Vol. 44, Elsevier, 445-487.

PETERSEN, S. A., R. D. FETTER, J. N. NOORDERMEER, C. S. GOODMAN AND A. DIANTONIO (1997). Genetic analysis of glutamate receptors in Drosophila reveals a retrograde signal regulating presynaptic transmitter release. Neuron 19: $1237-1248$.

PFEFFER SR (2017). Rab GTPases: master regulators that establish the secretory and endocytic pathways. Mol Biol Cell 28: 712-715

POTERYAEV, D., S. DATTA, K. ACKEMA, M. ZERIAL AND A. SPANG (2010). Identification of the switch in early-to-late endosome transition. Cell 141: 497-508.

STENMARK H (2009). Rab GTPases as coordinators of vesicle traffic. Nat Rev Mol Cell Biol 10: 513-525.

SON YE, JUNG WH, OH SH, KWAK JH, CARDENAS ME, PARK HS (2018). Mon1 Is Essential for Fungal Virulence and Stress Survival in Cryptococcus neoformans. Mycobiol. 46: 114-121.

STROUPE C (2018). This is the end: Regulation of Rab7 nucleotide binding in Endosomal Trafficking and Autophagy. Front.Cell Dev. Biol. 6: 129.

VERHOEVEN K, DE JONGHE P, COEN K, VERPOORTEN N, AUER-GRUMBACH M, KWON JM, FITZPATRICK D, SCHMEDDING E, DE VRIENDT E, JACOBS A VAN GERWEN V, WAGNER K, HARTUNG HP, TIMMERMAN V (2003). Mutations in the small GTP-ase late endosomal protein RAB7 cause Charcot-Marie-Tooth type 2B neuropathy. Am J Hum Genet 72: 722-727 
VERSTREKEN P, KOH TW, SCHULZE KL, ZHAI RG, HIESINGER PR, ZHOU Y, MEHTA SQ, CAO Y, ROOS J, BELLEN HJ (2003). Synaptojanin is recruited by endophilin to promote synaptic vesicle uncoating. Neuron 40: 733-748.

WANDINGER-NESSA, ZERIALM (2014). Rab proteins and the compartmentalization of the endosomal system. Cold Spring Harb Perspect Biol. 6: a022616.

WANG CW, STROMHAUG PE, SHIMA J, KLIONSKY DJ (2002). The Ccz1-Mon1 protein complex is required for the late step of multiple vacuole delivery pathways.
J Biol Chem 277: 47917-47927.

WANG CW, STROMHAUG PE, KAUFFMAN EJ, WEISMAN LS, KLIONSKY DJ (2003). Yeast homotypic vacuole fusion requires the Ccz1-Mon1 complex during the tethering/docking stage. J Cell Biol 163: 973-985

YOUSEFIAN, J., T. TROOST, F. GRAWE, T. SASAMURA, M. FORTINI et al.,2013). Dmon1 controls recruitment of Rab7 to maturing endosomes in Drosophila. $J$ Cell Sci 126: 1583-1594. 


\section{Further Related Reading, published previously in the Int. J. Dev. Biol.}

Spatially controlled expression of the Drosophila pseudouridine synthase RluA-1 Chung-Chi Wang, Jun-Chih Lo, Cheng-Ting Chien and Min-Lang Huang Int. J. Dev. Biol. (2011) 55: 223-227.

Patterning the nervous system through development and evolution Alain Ghysen, Christine Dambly-Chaudière and David W. Raible Int. J. Dev. Biol. (2010) 54: S1-S14.

Drosophila morgue influences cell numbers and positions in the embryonic nervous system

Barbara A. Schreader, Yiqin Wang, Stephanie Carter, Joanna Grigas and John R. Nambu Int. J. Dev. Biol. (2010) 54: 1425-1433.

Drosophila morgue influences cell numbers and positions in the embryonic nervous system

Barbara A. Schreader, Yiqin Wang, Stephanie Carter, Joanna Grigas and John R. Nambu Int. J. Dev. Biol. (2010) 54: 1425-1433.

Cell-cell signaling during neurogenesis: some answers and many questions. K M Bhat

Int. J. Dev. Biol. (1998) 42: 127-139.

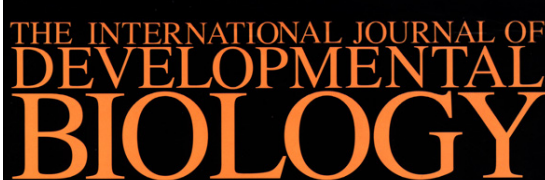

Volume 42 No. 3
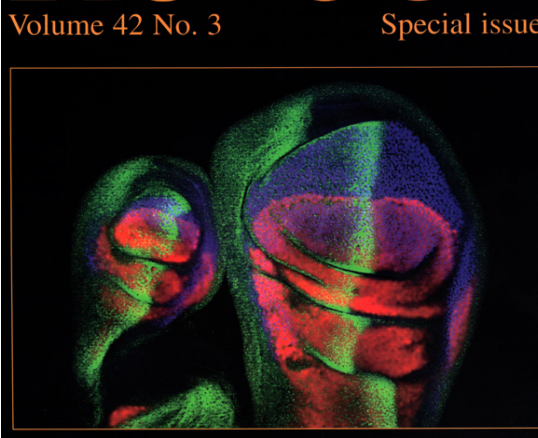

Developmental genetics of Drosophila

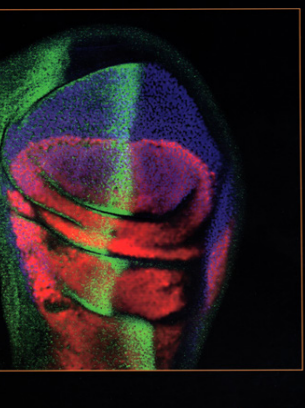

3

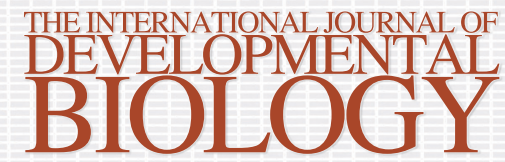

Volume 62 Nos. $11 / 12$

Special Issue

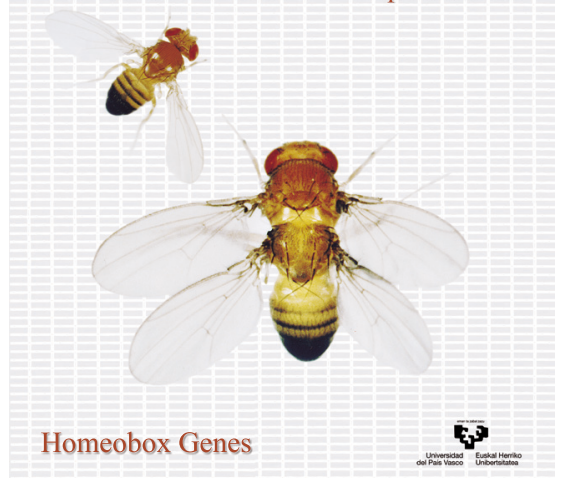

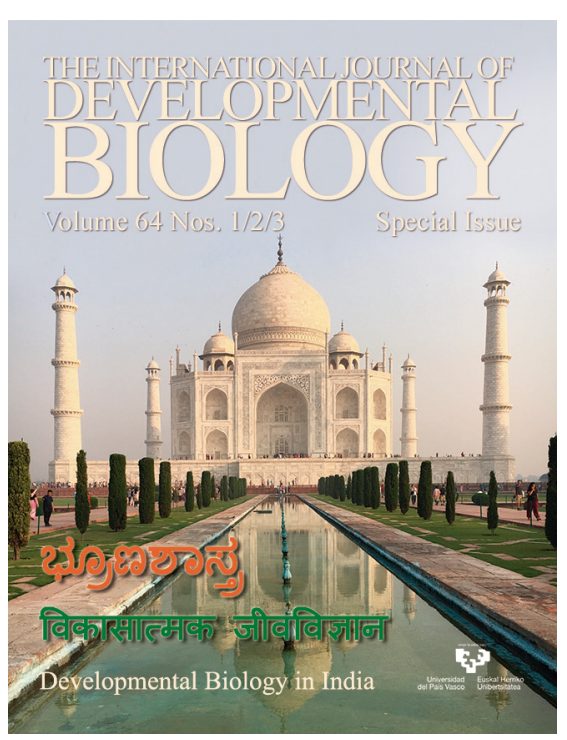

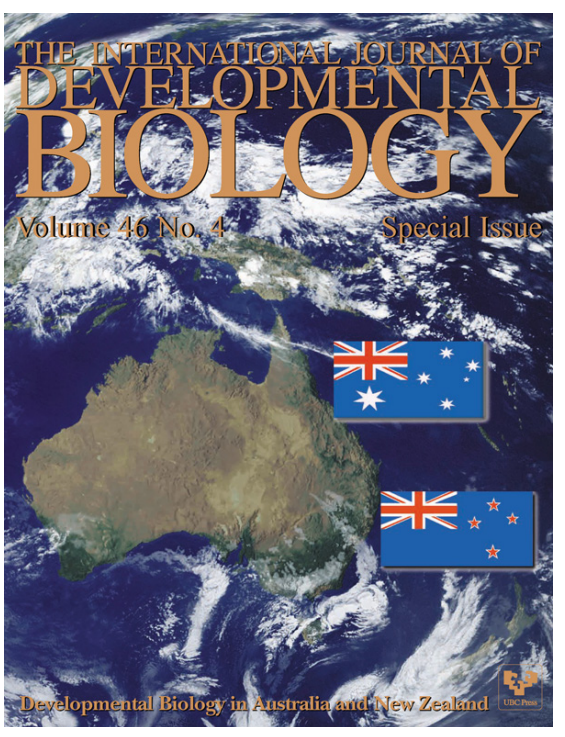

